\title{
Drawing Colored Graphs with Constrained Vertex Positions and Few Bends per Edge ${ }^{\star}$
}

\author{
Emilio Di Giacomo, Giuseppe Liotta, and Francesco Trotta
}

Dip. di Ingegneria Elettronica e dell'Informazione, Università degli Studi di Perugia

\{digiacomo, liotta, francesco.trotta\}@diei.unipg.it

\begin{abstract}
Hamiltonicity, book embeddability, and point-set embeddability of planar graphs are strictly related concepts. We exploit the interplay between these notions to describe colored sets of points and to design polynomial-time algorithms to embed $k$-colored planar graphs on these sets such that the resulting drawings have $\mathcal{O}(k)$ bends per edge.
\end{abstract}

\section{Introduction}

Let $G$ be a planar graph with $n$ vertices whose vertex set is partitioned into subsets $V_{0}, \ldots, V_{k-1}$ for some positive integer $1 \leq k \leq n$ and let $S$ be a set of $n$ distinct points in the plane partitioned into subsets $S_{0}, \ldots, S_{k-1}$ with $\left|V_{i}\right|=\left|S_{i}\right|$ $(0 \leq i \leq k-1)$. We say that each index $i$ is a color, $G$ is a $k$-colored planar graph, and $S$ is a $k$-colored set of points compatible with $G$. This paper studies the problem of computing a $k$-colored point-set embedding of $G$ on $S$, i.e. a crossing-free drawing of $G$ such that each vertex of $V_{i}$ is mapped to a distinct point of $S_{i}$. The problem has received considerable interest in the literature(see, e.g., $[13,5,6,8,9]$ ), also motivated by the observation that these types of drawings naturally model semantic constraints about the placement of the vertices. Particular attention has been devoted to the curve complexity of the computed drawings, i.e. the maximum number of bends along each edge. Namely, reducing the number of bends along the edges is a fundamental optimization goal when computing aesthetically pleasing drawings of graphs (see, e.g., 27]).

Two key references about $k$-colored point-set embeddings are the works by Kaufmann and Wiese [8] and by Pach and Wenger [9]. Kaufmann and Wiese 8] study the monochromatic version of the problem (i.e. the case when $k=1$ ) and prove that a planar graph with $n$ vertices always admits a point-set embedding with at most two bends per edge on any set of $n$ distinct points in the plane; they also proved that two bends per edge are necessary for some planar graphs and some configurations of points. Pach and Wenger [9] study the $n$-chromatic version of the problem and show that a linear number of bends is always sufficient to compute an $n$-colored point-set embedding of an $n$-colored planar graph $G$ on any $n$-colored set of points compatible with $G$; also they show that $\Omega(n)$

\footnotetext{
* Research partially supported by the MIUR Project "MAINSTREAM: Algorithms for massive information structures and data streams".
} 
bends per edge may be necessary even for $n$-colored paths when the points are in convex position.

The gap between constant curve complexity for $k=1$ and linear curve complexity for $k=n$ motivates the study of other values of $k$. In [5] it has been proved that there exists a 2-colored planar graph $G$ and a 2-colored set of points $S$ compatible with $G$ such that any 2 -colored points set embedding of $G$ on $S$ has at least one edge with $\Omega(n)$ bends. This result has been extended in [1, where it is proved that for $k$-colored point set embeddings such that $3 \leq k \leq n$, there may be cases requiring $\Omega(n)$ bends on $\Omega(n)$ edges. The two counterexamples presented in [5] and [1] are either tri-connected or have outerplanarity $\mathcal{O}(n)$, and thus a natural research direction is concerned with the curve complexity of $k$-colored point set embeddings for (sub)-families of planar graphs that have a simpler structure. In [3] it is proved that the curve complexity of 3-colored pointset embeddings may not be constant even for 3-colored outerplanar graphs.

These negative results suggest two different research directions, both devoted to studying $k$-colored point-set embeddings with curve complexity that does not depend on the input size. From one side, instead of restricting the classes of graphs to be drawn one can focus on special configurations of $k$-colored sets of points that make it possible to compute $k$-colored point-set embeddings with constant curve complexity for any $k$-colored planar graph. On the other side, one can ask what is the size of a universal $k$-colored set of points that guarantees curve complexity independent of $n$ for any $k$-colored planar graph. This last question can be asked both in the case that the points have real coordinates or by restricting them to form an integer grid. The main results in this paper can be outlined as follows.

- We study a special type of $k$-colored point-sets. Namely, let $G$ be any $k$ colored planar graph with $n$ vertices $(2 \leq k \leq n)$ and let $S$ be a $k$-colored set of $n$ points compatible with $G$. We show that if $S$ is ordered, i.e. for each color all points of that color are consecutive along the $x$-direction, then there exists an $\mathcal{O}(n \log n+k n)$-time algorithm that computes a $k$-colored point-set embedding of $G$ on $S$ with curve complexity at most $3 k+7$. This result generalizes to all $k$-colored planar graphs a similar result presented in 3 . for $k$-colored outerplanar graphs and makes it possible to improve a related result of [1].

- We show the existence of $k$-colored sets of points having linear size and supporting $k$-colored point-set embeddings of $\mathcal{O}(k)$ curve complexity. Namely, let $\mathcal{F}_{k}$ be the family of all $k$-colored planar graphs with $n$ vertices $(1 \leq k \leq n)$. For any $G \in \mathcal{F}_{k}$ and for any $k$-colored set of points $S$ such that $S$ contains $k n-k^{2}+1$ points for each color, there exists an $\mathcal{O}(n \log n+k n)$-time algorithm that computes a $k$-colored point-set embedding of $G$ on $S$ with curve complexity at most $3 k+7$. We recall that, even for 2 -colored simple paths, a universal 2 colored set of points that supports straight-line 2-colored point-set embeddings may need a quadratic number of points [6].

- Since the above result implies a total number of $k^{2} n-k^{3}+k$ points in $S$, one can ask whether $n+o(n)$ points are sufficient to guarantee a curve complexity that does not depend on $n$. We give a negative answer to this question for $k=2$. 
Namely, let $c$ be any constant such that $c>1$. We prove that for $k=2$ there exists a set $S$ of $n+\frac{n}{c}$ points and a 2-colored planar graph $G$ such that any 2-colored point set embedding of $G$ on $S$ has an edge requiring at least $\Omega(n)$ bends.

- Finally, we show that every $k$-colored planar graph with $n$ vertices admits a $k$-colored points-set embedding with curve complexity $6 k+5$ on a $k$-colored grid whose size is $\mathcal{O}\left(k n^{2}\right) \times \mathcal{O}\left(k n^{2}\right)$. Such $k$-colored points-set embedding can be computed in $\mathcal{O}(k n)$ time.

The above results are all based on a novel approach to the problem of computing $k$-colored point-set embeddings of planar graphs. Namely we exploit the notion of simultaneous $k$-colored book embedding of a $k$-colored planar graph and a $k$-colored path and show how this notion can be used to compute a suitable Hamiltonian circuit on the graph; in turn, we use the Hamiltonian circuit to compute a point-set embedding with $\mathcal{O}(k)$ curve complexity. For reasons of space some proofs have been sketched or omitted.

\section{Preliminaries}

Let $G=(V, E)$ be a graph. A $k$-coloring of $G$ is a partition $\left\{V_{0}, V_{1}, \ldots, V_{k-1}\right\}$ of $V$ where the integers $0,1, \ldots, k-1$ are called colors. In the rest of this section the index $i$ is $0 \leq i \leq k-1$ if not differently specified. For each vertex $v \in V_{i}$ we denote by $\operatorname{col}(v)$ the color $i$ of $v$. A graph $G$ with a $k$-coloring is called a $k$-colored graph. Let $S$ be a set of distinct points in the plane. We always assume that the points of $S$ have distinct $x$-coordinates (this condition can always be satisfied by means of a suitable rotation of the plane). For any point $p$ in the Euclidean plane we denote by $x(p)$ and $y(p)$ the $x$ - and $y$-coordinates of $p$, respectively. A $k$-coloring of $S$ is a partition $\left\{S_{0}, S_{1}, \ldots, S_{k-1}\right\}$ of $S$. A set $S$ of distinct points in the plane with a $k$-coloring is called a $k$-colored set of points. For each point $p \in S_{i} \operatorname{col}(p)$ denotes the color $i$ of $p$. A $k$-colored set of points $S$ is compatible with a $k$-colored graph $G$ if $\left|V_{i}\right|=\left|S_{i}\right|$ for every $i$. Let $G$ be planar. We say that $G$ has a $k$-colored point-set embedding on $S$ if there exists a planar drawing of $G$ such that: (i) every vertex $v$ is mapped to a distinct point $p$ of $S$ with $\operatorname{col}(p)=\operatorname{col}(v)$, (ii) each edge $e$ of $G$ is drawn as a polyline; a point shared by any two consecutive segments of the polyline is called a bend of $e$. The curve complexity of a drawing is the maximum number of bends per edge. Given a vertex $v$ of $G$ we denote by $p_{v}$ the point representing $v$ in the drawing. A $k$ colored sequence $\sigma$ is a linear sequence of (possibly repeated) colors $c_{0}, c_{1}, \ldots$, $c_{n-1}$ such that $0 \leq c_{j} \leq k-1(0 \leq j \leq n-1)$. We say that $\sigma$ is compatible with a $k$-colored graph $G$ if, for every $i$ color $i$ occurs $\left|V_{i}\right|$ times in $\sigma$. Let $S$ be a $k$ colored set of points and let $p_{0}, p_{1}, \ldots, p_{n-1}$ be the points of $S$ ordered according to their $x$-coordinates. Let $P=\left(v_{0}, v_{1}, \ldots, v_{n-1}\right)$ be a path with $n$ vertices such that $c\left(v_{i}\right)=c\left(p_{i}\right)$. We say that $P$ is the path induced by $S$ and denote it as $\operatorname{path}(S)$. We also say that $\sigma=c\left(p_{0}\right), c\left(p_{1}\right), \ldots, c\left(p_{n-1}\right)$ is the $k$-colored sequence induced by $S$ and denote it as $\operatorname{seq}(S)$.

A graph $G$ has a Hamiltonian path if it has a simple path that contains all the vertices of $G$. $G$ has a Hamiltonian cycle if it has a simple cycle that contains all 
the vertices of $G$. If $G$ is a $k$-colored graph and $\sigma=c_{0}, c_{1}, \ldots, c_{n-1}$ is a $k$-colored sequence compatible with $G$, a $k$-colored Hamiltonian path of $G$ consistent with $\sigma$ is a Hamiltonian path $v_{0}, v_{1}, \ldots, v_{n-1}$ such that $\operatorname{col}\left(v_{i}\right)=c_{i}(0 \leq i \leq n-1)$. A $k$-colored Hamiltonian cycle of $G$ consistent with $\sigma$ is a Hamiltonian cycle $v_{0}, v_{1}, \ldots, v_{n-1}$ such that $\operatorname{col}\left(v_{i}\right)=c_{i}(0 \leq i \leq n-1)$. A $k$-colored planar graph $G$ can always be augmented to a (not necessarily planar) $k$-colored graph $G^{\prime}$ by adding to $G$ a suitable number of dummy edges and such that $G^{\prime}$ has a $k$-colored Hamiltonian cycle $\mathcal{C}$ consistent with $\sigma$ and that includes all dummy edges. If $G^{\prime}$ is not planar, we can apply a planarization algorithm (see, e.g., [2]) to $G^{\prime}$ with the constraint that only crossings between dummy edges and edges of $G-\mathcal{C}$ are allowed. Such a planarization algorithm constructs an embedded planar graph $G^{\prime \prime}$ where each edge crossing is replaced with a dummy vertex, called division vertex. By this procedure each edge $e$ of $\mathcal{C}$ can be transformed into a path whose internal vertices are division vertices: let $\mathcal{C}^{\prime}$ be the resulting cycle. Let $e$ be an edge of $\mathcal{C}^{\prime}$ (notice that the endvertices of $e$ are either vertices of $G$ or division vertices). The path $\mathcal{H}=\mathcal{C}^{\prime} \backslash e$ is called an augmenting $k$-colored Hamiltonian path of $G$ consistent with $\sigma$. The graph $G^{\prime \prime} \backslash e$ is called the augmented Hamiltonian form of $G$ and is denoted as $\operatorname{Ham}(G)$. If every edge $e$ of $G$ is crossed at most $d$ times in $G^{\prime}$ (which implies that $e$ is split by at most $d$ division vertices in $\operatorname{Ham}(G)), \mathcal{H}$ is said to be an augmenting $k$-colored Hamiltonian path of $G$ consistent with $\sigma$ and inducing at most $d$ division vertices per edge. If $G^{\prime}$ is planar, then $\operatorname{Ham}(G)=G^{\prime}$ and $\mathcal{H}$ is defined as $\mathcal{C} \backslash e$, where $e$ is any edge of $\mathcal{C}$. Notice that the endvertices of $\mathcal{H}$ are on the same face $f$ of $\operatorname{Ham}(G)$; we may assume that $f$ is the external face (if not we can choose an embedding of $\operatorname{Ham}(G)$ such that $f$ is the external face).

Let $v_{d}$ be a division vertex for an edge $e$ of $G$. Since a division vertex corresponds to a crossing between $e$ and an edge of $\mathcal{C}$, there are four edges incident on $v_{d}$ in $G^{\prime \prime}$; two of them are dummy edges that belong to $\mathcal{C}^{\prime}$, the other two are two "pieces" of edge $e$ obtained by splitting $e$ with $v_{d}$. Let $\left(u, v_{d}\right)$ and $\left(v, v_{d}\right)$ be the latter two edges. We say that $v_{d}$ is a flat division vertex if it is encountered after $u$ and before $v$ while walking along $\mathcal{H} ; v_{d}$ is a pointy division vertex otherwise. Notice that there are exactly four edges incident on $v_{d}$ in $G^{\prime \prime}$, but there can be only three edges incident on $v_{d}$ in $\operatorname{Ham}(G)$ (this happens if the edge removed from $G^{\prime \prime}$ to obtain $\operatorname{Ham}(G)$ has $v_{d}$ as an endvertex, i.e. if $v_{d}$ is one of the two endvertices of $\mathcal{H}$ ). However the edge incident on $v_{d}$ that is removed is neither $\left(u, v_{d}\right)$, nor $\left(v, v_{d}\right)$ because the removed edge is an edge of $\mathcal{C}^{\prime}$. It follows that the definition of flat and pointy division vertex apply to $v_{d}$ also in the case when $v_{d}$ is an endvertex of $\mathcal{H}$. The following theorem has been proved in [1].

Theorem 1. 1] Let $G$ be a $k$-colored planar graph, let $\sigma$ be a $k$-colored sequence compatible with $G$, and let $\mathcal{H}$ be an augmenting $k$-colored Hamiltonian path of $G$ compatible with $\sigma$ inducing at most d division vertices per edge, $d_{p}$ of which are pointy division vertices. Then $G$ admits a $k$-colored point set embedding $\Gamma$ on any set of points that induces $\sigma$ such that the curve complexity is $d+d_{p}+1$. Furthermore, there exists an $\mathcal{O}(n \log n)$-time algorithm that computes $\Gamma$. 
A spine is an horizontal line. Let $\ell$ be a spine and let $p, q$ be two points of $\ell$. An arc is a circular arc passing through $p$ and $q$. We say that the arc is in the top (bottom) page if it belongs to the half plane above (below) the spine. Let $G=(V, E)$ be a planar graph. A topological book embedding of $G$ is a planar drawing such that all vertices of $G$ are represented as points of a spine $\ell$ and each edge can be either above the spine, or below the spine, or it can cross the spine. Each crossing between an edge and the spine is called a spine crossing. It is also assumed that in a topological book embedding every edge consists of one or more arcs such that no two consecutive arcs are in the same page. An edge $e$ is said to be in the top (bottom) page of the spine if it consists of exactly one arc and this arc is in the top (bottom) page. A monotone topological book embedding is a topological book embedding such that each edge crosses the spine at most once. Also, let $e=(u, v)$ be an edge of a monotone topological book embedding that crosses the spine at a point $p$; $e$ is such that if $u$ precedes $v$ in the left-to-right order along the spine then $p$ is between $u$ and $v$, the arc with endpoints $u$ and $p$ is in the bottom page, and the arc with endpoints $u$ and $v$ is in the top page. The edges that do not cross the spine are called $u$-shaped edges, while edges that cross the spine are called $s$-shaped edges. The following theorem has been proved in [4].

Theorem 2. 4] Every planar graph admits a monotone topological book embedding. Also, a monotone topological book embedding can be computed in $\mathcal{O}(n)$ time, where $n$ is the number of the vertices in the graph.

Let $\Gamma$ be a topological book emebedding of a planar graph $G$. A point $p$ of the spine $\ell$ of $\Gamma$ is accessible from the top (bottom) page of $\Gamma$ if the vertical half-line $\ell^{\prime}$ starting at $p$ that is in the half-plane above (below) $\ell$ does not cross any arc of $\Gamma$. If $\ell^{\prime}$ crosses an arc $a$, we say that a covers $p$. The local top (bottom) page width of $\Gamma$ on $p \operatorname{lw}_{\mathrm{t}}(\Gamma, p)\left(\operatorname{lw}_{\mathrm{b}}(\Gamma, p)\right)$ is the number of arcs in the top (bottom) page of $\Gamma$ that cover $p$. The cumulative local page width of $\Gamma$ on $p$ is $\operatorname{clw}(\Gamma, p)=$ $\operatorname{lw}_{\mathrm{t}}(\Gamma, p)+\operatorname{lw}_{\mathrm{b}}(\Gamma, p)$. The cumulative width of $\Gamma$ is $\mathrm{cw}(\Gamma)=\max _{p \in l}\{\operatorname{clw}(\Gamma, p)\}$. The top page width of $\Gamma$ is $\mathrm{w}_{\mathrm{t}}(\Gamma)=\max _{p \in l}\left\{\operatorname{lw}_{\mathrm{t}}(\Gamma, p)\right\}$, and analogously the bottom page width of $\Gamma$ is $\mathrm{w}_{\mathrm{b}}(\Gamma)=\max _{p \in l}\left\{\operatorname{lw}_{\mathrm{b}}(\Gamma, p)\right\}$. Finally the width of $\Gamma$ is $\mathrm{w}(\Gamma)=\max \left\{\mathrm{w}_{\mathrm{t}}(\Gamma), \mathrm{w}_{\mathrm{b}}(\Gamma)\right\}$. Notice that the following inequality is always satisfied $\mathrm{w}(\Gamma) \leq \mathrm{cw}(\Gamma) \leq \mathrm{w}_{\mathrm{t}}(\Gamma)+\mathrm{w}_{\mathrm{b}}(\Gamma) \leq 2 \mathrm{w}(\Gamma)$.

\section{Overview of the Approach}

In this section we give a high-level description of the approach followed throughout the paper. We need some additional definitions. Let $\Gamma$ be a topological book embedding of a $k$-colored planar graph $G$ and let $v_{0}, v_{1}, \ldots, v_{n-1}$ be the vertices of $G$ in the order they appear along the spine of $\Gamma$. The $k$-colored sequence $c\left(v_{0}\right), c\left(v_{1}\right), \ldots, c\left(v_{n-1}\right)$ is called the $k$-colored sequence induced by $\Gamma$. Let $P$ be a path and let $v_{0}, v_{1}, \ldots, v_{n-1}$ be the vertices of $P$ in the order they appear along $P$; the $k$-colored sequence $c\left(v_{0}\right), c\left(v_{1}\right), \ldots, c\left(v_{n-1}\right)$ is called the $k$-colored sequence induced by $P$. Let $\Gamma_{P}$ be a topological book embedding of $P . \Gamma_{P}$ is 
external if both the endvertices of $P$ are accessible either from the top page or from the bottom page. Let $G_{1}=\left(\bigcup_{i=0}^{k-1} V_{1, i}, E_{1}\right)$ and $G_{2}=\left(\bigcup_{i=0}^{k-1} V_{2, i}, E_{2}\right)$ be two planar $k$-colored graphs. We say that $G_{1}$ and $G_{2}$ are compatible if $\left|V_{1, i}\right|=\left|V_{2, i}\right|$ for every $i=0, \ldots, k-1$. A simultaneous $k$-colored book embedding of two compatible planar graphs $G_{1}$ and $G_{2}$ is a pair of drawings $<\Gamma_{1}, \Gamma_{2}>$ such that: (i) $\Gamma_{i}$ is a topological book embedding $(i=1,2)$; (ii) $\Gamma_{1}$ and $\Gamma_{2}$ use the same points to represent the vertices; and (iii) the $k$-colored sequences induced by $\Gamma_{1}$ and $\Gamma_{2}$ coincide. We are now ready to describe our approach:

Step 1: Let $P=\operatorname{path}(S)$. Compute a simultaneous $k$-colored book embedding $<\Gamma_{G}, \Gamma_{P}>$ of $G$ and $P$, such that $\Gamma_{G}$ is a monotone topological book embedding of $G$ and $\Gamma_{P}$ is external;

Step 2: By using $\left\langle\Gamma_{G}, \Gamma_{P}\right\rangle$, compute a $k$-colored point-set embedding of $G$ on $S$. The curve complexity of the computed drawing is bounded by the width of $\Gamma_{P}$.

The idea behind the above described approach is based on Theorem 1 and on the following lemma, that shows how to compute an augmenting $k$-colored Hamiltonian path by using $\left\langle\Gamma_{G}, \Gamma_{P}\right\rangle$. An illustration of such an idea is shown in Figure 1.

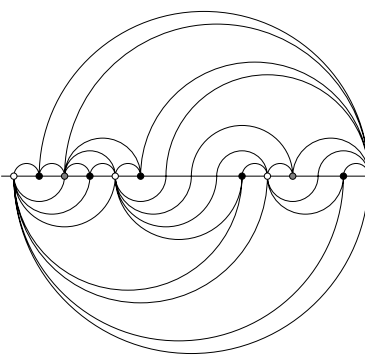

(a)

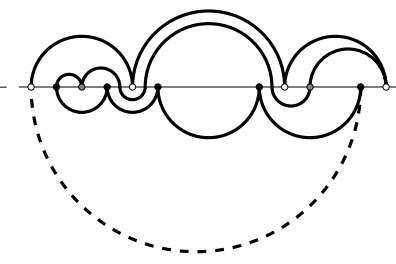

(b)

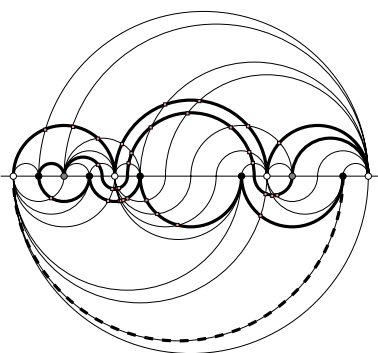

(c)

Fig. 1. (a) A monotone topological book embedding $\Gamma_{G}$ of a planar 3-colored graph $G$. (b) An external topological book embedding $\Gamma_{P}$ of a path $P$. Notice that $\left\langle\Gamma_{G}, \Gamma_{P}\right\rangle$ is a simultaneous $k$-colored book embedding of $G$ and $P$ and that $\mathrm{w}\left(\Gamma_{P}\right)=3$. (c) An augmenting $k$-colored Hamiltonian path of $G$ consistent with the $k$-colored sequence induced by $P$ and inducing at most 5 division vertices per edge.

Lemma 1. Let $G$ and $P$ be a $k$-colored planar graph and a $k$-colored path that are compatible. Let $\left\langle\Gamma_{G}, \Gamma_{P}\right\rangle$ be a simultaneous $k$-colored book embedding of $G$ and $P$, such that $\Gamma_{G}$ is a monotone topological book embedding and $\Gamma_{P}$ is external. Let $\sigma$ be the $k$-colored sequence induced by $P$. Then $G$ admits an augmenting $k$-colored Hamiltonian path consistent with $\sigma$ that induces at most $2 \mathrm{w}\left(\Gamma_{P}\right)+\mathrm{cw}\left(\Gamma_{P}\right)+2$ division vertices for every s-shaped edge of $\Gamma_{G}$ and at most $2 \mathrm{w}\left(\Gamma_{P}\right)+1$ division vertices for every $u$-shaped edge of $\Gamma_{G}$. 
Sketch of Proof: Consider the simultaneous $k$-colored book embedding $\left\langle\Gamma_{G}, \Gamma_{P}\right\rangle$. Since $\Gamma_{P}$ is external the two endvertices $u$ and $v$ of $P$ are accessible either from the top page or from the bottom page. Vertices $u$ and $v$ can be connected by means of an edge $e^{\prime}$ consisting of at most two arcs. Namely, if they are both accessible from the same page, say the top one, then we connect them with an arc in the top page; if they are accessible from different pages, assume that $u$ is accessible from the top page and $v$ is accessible from the bottom page (the other case is symmetric). We create an arc connecting $v$ to a point $p$ of the spine that is to the right of the rightmost point (vertex or spine crossing) of $\Gamma_{P}$; then we add an arc connecting $p$ to $u . \Gamma^{\prime}=\Gamma_{G} \cup \Gamma_{P} \cup e^{\prime}$ is a (possibly non-planar) drawing of a (possibly non-planar) graph $G^{\prime}$ such that $\mathcal{C}=P \cup e^{\prime}$ is a $k$-colored Hamiltonian cycle consistent with the $k$-colored sequence $\sigma$ induced by $P$. If $G^{\prime}$ is not planar, since both $\Gamma_{G}$ and $\Gamma_{P}$ are planar, a crossing in $\Gamma^{\prime}$ is possible only between the edges of $\Gamma_{G}$ and the edges of $\Gamma_{P}$. We replace each crossing with a division vertex. It may happen that edge $e^{\prime}$ is also subdivided (this happens if the endvertices of $P$ are not on the same face of $\Gamma_{G}$ ). In all cases, there exists a portion of $e^{\prime}$ that is contained in a face of $\Gamma_{G}$. The graph obtained by removing this portion (possibly coincident with $e^{\prime}$ itself) is the augmented Hamiltonian form of $G$. The concatenation of $P$ with the portion of $e^{\prime}$ that is not removed forms an augmenting $k$-colored Hamiltonian path of $G$ consistent with the $k$-colored sequence induced by $P$. In order to compute the number of division vertices on each edge of $G$, we first count the number of crossings between an edge of $G$ and the edges of $P$, and then we count the extra division vertices introduced when adding edge $e^{\prime}$. Let $e=(u, v)$ be an $u$-shaped edge of $\Gamma_{G}$. Assume that $e$ is in the top page of $\Gamma_{G}$. The number of crossings between $e$ and the edges of $P$ is $c=\operatorname{lw}_{\mathrm{t}}\left(\Gamma_{P}, p_{u}\right)+\operatorname{lw}_{\mathrm{t}}\left(\Gamma_{P}, p_{v}\right) \leq 2 \mathrm{w}\left(\Gamma_{P}\right)$. Let $e=(u, v)$ be an $s$-shaped edge and let $a_{1}$ and $a_{2}$ be the two arcs that form $e$. Arc $a_{1}$ has $p_{u}$ and $d$ as its endpoints, where $d$ is the point where $e$ crosses the spine. Arc $a_{2}$ has $d$ and $p_{v}$ as its endpoints. The number of crossings between $e$ and the edges of $P$ is $c=\operatorname{lw}_{\mathrm{t}}\left(\Gamma_{P}, p_{u}\right)+\operatorname{clw}\left(\Gamma_{P}, d\right)+\operatorname{lw}_{\mathrm{b}}\left(\Gamma_{P}, p_{v}\right) \leq \mathrm{w}\left(\Gamma_{P}\right)+\mathrm{cw}\left(\Gamma_{P}\right)+\mathrm{w}\left(\Gamma_{P}\right)=$ $2 \mathrm{w}\left(\Gamma_{P}\right)+\mathrm{cw}\left(\Gamma_{P}\right)$. Since $e^{\prime}$ consists of at most two arcs in different pages, each arc of $\Gamma_{G}$ can have one additional division vertex caused by the addition of $e^{\prime}$. Therefore an $u$-shaped edge ( $s$-shaped edge) can have at most $2 \mathrm{w}\left(\Gamma_{P}\right)+1$ $\left(2 \mathrm{w}\left(\Gamma_{P}\right)+\mathrm{cw}\left(\Gamma_{P}\right)+2\right)$ division vertices per edge.

\section{Ordered $k$-Colorings}

Let $S$ be a $k$-colored set of points such that for every pair of points $p_{1}$ and $p_{2}$ with the same color, there is no point $q$ such that $x\left(p_{1}\right)<x(q)<x\left(p_{2}\right)$ and $c(q) \neq c\left(p_{1}\right)=c\left(p_{2}\right)$. We say that $S$ is an ordered $k$-colored set of points. In other words, an ordered $k$-colored set of points is such that all points of each color are consecutive according to the $x$-coordinate ordering. Analogously, we define an ordered $k$-colored path $P$ to be a path where all vertices of the same color appear consecutively walking along $P$, and an ordered $k$-colored sequence to be a $k$ colored sequence where all elements with the same color appear consecutively 
in the sequence. The technique behind this proof is a variant of the algorithm used in [3] to compute an augmenting $k$-colored Hamiltonian cycle of a $k$-colored simple cycle.

Lemma 2. Let $G$ and $P$ be a planar $k$-colored graph and an ordered $k$-colored path that are compatible. There exists a simultaneous $k$-colored book embedding $<\Gamma_{G}, \Gamma_{P}>$ of $G$ and $P$, such that $\Gamma_{G}$ is a monotone topological book embedding, $\Gamma_{P}$ is external and $\mathrm{cw}\left(\Gamma_{P}\right) \leq k$. Furthermore, $\left\langle\Gamma_{G}, \Gamma_{P}\right\rangle$ can be computed in $\mathcal{O}(k n)$ time.

Theorem 3. Let $G$ be a planar $k$-colored graph, and let $\sigma$ be an ordered $k$ colored sequence. Then $G$ admits an augmenting $k$-colored Hamiltonian path $\mathcal{H}$ consistent with $\sigma$ that induces at most $3 k+2$ division vertices per edge; at most 4 of these are pointy division vertices. Furthermore, $\mathcal{H}$ can be computed in $\mathcal{O}(k n)$ time.

Sketch of Proof: Let $P$ be an ordered $k$-colored path that is compatible with $G$ and such that the $k$-colored sequence induced by $P$ coincides with $\sigma$. By Lemma 1. $G$ admits an augmenting $k$-colored Hamiltonian path $\mathcal{H}$ consistent with $\sigma$ and inducing at most $2 \mathrm{w}\left(\Gamma_{P}\right)+\mathrm{cw}\left(\Gamma_{P}\right)+2$ division vertices for every $s$ shaped edge of $\Gamma_{G}$ and at most $2 \mathrm{w}\left(\Gamma_{P}\right)+1$ division vertices for every $u$-shaped edge of $\Gamma_{G}$. Since by Lemma $2 \mathrm{cw}\left(\Gamma_{P}\right) \leq k$ and since $\mathrm{w}\left(\Gamma_{P}\right) \leq \mathrm{cw}\left(\Gamma_{P}\right)$, it follows that $\mathcal{H}$ induces at most $3 k+2$ division vertices for every $s$-shaped edge and at most $2 k+1$ division vertices for every $u$-shaped edge. We now count the number of pointy division vertices. We first recall (see Lemma 1) that an edge can have one or two division vertices caused by the addition of an edge $e^{\prime}$ that transforms $P$ into a cycle. Such division vertices are necessarily pointy division vertices. Namely, since a portion of $e^{\prime}$ is removed to obtain $\mathcal{H}$, then all the division vertices caused by the addition of $e^{\prime}$ appear at the beginning of $\mathcal{H}$ or at its end. Let $V_{d}^{\prime}$ be the set of these division vertices and let $v_{d}$ be one of them. Let $\left(u^{\prime}, v_{d}\right)$ and $\left(v_{d}, v^{\prime}\right)$ be the two edges incident to $v_{d}$ that are not in $\mathcal{H}$. Vertices (either real or division vertices) $u^{\prime}$ and $v^{\prime}$ are not in $V_{d}^{\prime}$ and therefore they are encountered both after $v_{d}$ or both before $v_{d}$ when walking along $\mathcal{H}^{\prime}$, i.e. $v_{d}$ is a pointy division vertex. Concerning the division vertices that are not created by the addition of $e^{\prime}$, it can be proved that at most one, in the case of $u$-shaped edges, or two, in the case of $s$-shaped edges, of these division vertices are pointy. It follows that an $u$-shaped edge can have at most two pointy division vertices and an $s$-shaped edge can have at most four pointy division vertices.

A consequence of Theorem 3 and Theorem 1 is the following.

Theorem 4. Let $G$ be a $k$-colored planar graph with $n$ vertices and let $S$ be an ordered $k$-colored set of points compatible with $G$. There exists an $\mathcal{O}(n \log n+$ $k n$ )-time algorithm that computes a $k$-colored point-set embedding of $G$ on $S$ having curve complexity at most $3 k+7$.

Theorem 3 can be applied also to another special $k$-coloring. Namely, let $G=$ $\left(\bigcup_{i=0}^{k-1} V_{i}, E\right)$ be a $k$-colored planar graph; we say that the coloring of $G$ is an 
unbalanced $k$-colorings if $\left|V_{i}\right|=1(i=0,1, \ldots, k-2)$ and $\left|V_{k-1}\right|=n-k+1$. In [1] it has been proved that if a $k$-colored planar graph $G$ has an unbalanced $k$-colorings, then $G$ admits a $k$-colored point-set embedding on any given set of points compatible with $G$ with curve complexity at most $9 k-1$. We use Theorem 3 to improve this bound.

Theorem 5. Let $G$ be an $n$-vertex $k$-colored planar graph with an unbalanced coloring and let $S$ be a $k$-colored set of points compatible with $G$. There exists an $\mathcal{O}(n \log n+k n)$-time algorithm that computes a $k$-colored point-set embedding of $G$ on $S$ having curve complexity at most $6 k+4$.

\section{$5 \quad h$-Bend $k$-Colored Universal Sets and Grids}

Let $\mathcal{F}$ be a family of $k$-colored planar graphs such that every element of $\mathcal{F}$ has $n$ vertices and $1 \leq k \leq n$; let $S$ be a $k$-colored set of points. We say that $S$ is an $h$-bend $k$-colored universal set for $\mathcal{F}$ if, for every $G \in \mathcal{F}, G$ has a $k$-colored pointset embedding on $S$ having at most $h$ bends per edge. In this section we shall use Theorem 3 to describe $h$-bend $k$-colored universal sets of points that can either have real coordinates (Subsection 5.1) or form an integer grid (Subsection 5.2).

\section{$5.1 h$-Bend $k$-Colored Universal Sets}

Let $\mathcal{F}_{k}$ be the family of all $k$-colored planar graphs with $n$ vertices $(1 \leq k \leq n)$. In this section we show that there exist $h$-bend $k$-colored universal sets for $\mathcal{F}_{k}$ such that the number of points in the sets is $O(n)$ and $h$ does not depend on $n$; we also show a lower bound on the size of such sets for the family $\mathcal{F}_{2}$. We start with a lemma that shows an $h$-bend $k$-colored universal set for a sub-family of all $k$-colored planar graphs with $n$ vertices. Let $\mathcal{F}_{k}^{\prime}$ be the family of $k$-colored planar graphs such that every graph of $\mathcal{F}_{k}^{\prime}$ has $n$ vertices and every two graphs of the family have the same number of vertices with color $i(1 \leq i \leq k)$. It is known that every $k$-colored set $S$ of $n$ points compatible with the graphs in $\mathcal{F}_{k}^{\prime}$ is an $h$-bend $k$-colored universal set for $\mathcal{F}_{k}^{\prime}$ with $h=\mathcal{O}(n)$ [19]. The next lemma shows that by adding $O(n)$ extra points to $S$, the curve complexity can become independent of $n$.

Lemma 3. Let $G=\left(\bigcup_{i=0}^{k-1} V_{i}, E\right)$ be a $k$-colored planar graph with $n$ vertices $(1 \leq k \leq n)$ with $\left|V_{i}\right|=n_{i}(i=0,1, \ldots, k-1)$; let $S=\bigcup_{i=0}^{k-1} S_{i}$ be any $k$-colored set of points such that $\left|S_{i}\right|=k\left(n_{i}-1\right)+1$. There exists an $\mathcal{O}(n \log n+k n)$-time algorithm that computes a $k$-colored point-set embedding of $G$ on $S$ having curve complexity at most $3 k+7$.

Sketch of Proof: We prove the statement by showing that it is possible to remove $(k-1)\left(n_{i}-1\right)$ points from $S_{i}(i=0,1, \ldots, k-1)$ in such a way that the remaining $n=\sum_{i=0}^{k-1} n_{i}$ points form a set $S^{\prime}$ which induces an ordered $k$-colored sequence compatible with $G$. This, along with Theorem 3 , implies that $G$ admits an augmenting $k$-colored Hamiltonian path consistent with $\sigma=\operatorname{seq}\left(S^{\prime}\right)$ that 
induces at most $3 k+2$ division vertices; at most 4 of these division vertices are pointy division vertices. Such an augmenting $k$-colored Hamiltonian path can be computed in $\mathcal{O}(k n)$. By Theorem 1, there exists an $\mathcal{O}(n \log n)$-time algorithm that computes a $k$-colored point-set embedding of $G$ on $S^{\prime}$ having curve complexity at most $3 k+7$. Since there is a one-to-one mapping between the points of $S$ and the elements of $\sigma_{k}=\operatorname{seq}(S)$, in the rest of this proof we concentrate on the sequence $\sigma_{k}$ and prove that elements can be removed from $\sigma_{k}$ in order to obtain an ordered $k$-colored sequence $\sigma_{k}^{\prime}$ compatible with $G$. More precisely, we prove that given a $k$-colored sequence $\sigma_{k}$ such that the number of elements colored $i$ is at least $k\left(n_{i}-1\right)+1$, it is possible to remove some elements from $\sigma_{k}$ in order to create an ordered $k$-colored sequence $\sigma_{k}^{\prime}$ compatible with $G$. The proof is by induction on the number of colors $k$. If $k=1$ it is sufficient to arbitrarily remove $(k-1)\left(n_{0}-1\right)$ points (i.e. to remove no point) and the obtained sequence is an ordered 1-colored sequence compatible with $G$. If $k>1$, let $\sigma_{k}=c_{0}, \ldots, c_{k(n-k+1)-1}$. We denote as $\sigma_{i, j}$ the subsequence $c_{i}, c_{i+1}, \ldots, c_{j}$ of $\sigma_{k}$. Let $j_{i}=\min \left\{j \mid \sigma_{0, j}\right.$ contains $n_{i}$ elements whose value is $\left.i\right\}$ for $i=0, \ldots, k-1$ and let $j=\min _{i}\left\{j_{i}\right\}$. Without loss of generality, assume that $j=j_{0}$. The sequence $\sigma_{0, j}$ contains $n_{0}$ elements whose value is 0 and at most $n_{i}-1$ elements whose value is $i(i=1,2, \ldots, k-1)$. Therefore $\sigma_{j+1, k(n-k+1)-1}$ contains at least $(k-1)\left(n_{i}-1\right)+1$ elements whose value is $i(i=1,2, \ldots, k-1)$. The sequence $\sigma_{(k-1)}=\sigma_{j+1, k(n-k+1)-1} \backslash\left\{c_{j} \mid c_{j}=0\right\}$ is a $(k-1)$-colored sequence such that the number of elements colored $i$ is at least $(k-1)\left(n_{i}-1\right)+1$. Thus, by induction, one can remove elements from $\sigma_{(k-1)}$ in order to obtain an ordered $(k-1)$-colored sequence $\sigma_{k-1}^{\prime}$ compatible with $G \backslash\{v \in V \mid \operatorname{col}(v)=0\}$. It follows that the sequence $\sigma_{k}^{\prime}=\sigma_{0, j} \backslash\left\{c_{j} \mid c_{j} \neq 0\right\} \cup \sigma_{k-1}^{\prime}$ is an ordered $k$-colored sequence compatible with $G$.

Theorem 6. Let $\mathcal{F}_{k}$ be the family of all $k$-colored planar graphs with $n$ vertices $(1 \leq k \leq n)$. Any $k$-colored set of points $S$ such that $S$ contains $k n-k^{2}+1$ points for each color is a $(3 k+7)$-bend $k$-universal set for $\mathcal{F}_{k}$. Furthermore, there exists an $\mathcal{O}(n \log n+k n)$-time algorithm that computes a $k$-colored point-set embedding on $S$ of any $G \in \mathcal{F}_{k}$ with curve complexity at most $3 k+7$.

Sketch of Proof: Let $G$ be a graph of $\mathcal{F}_{k}$. For each color $i, G$ has at most $n-k+1$ vertices of color $i$. Since $S$ has $k(n-k)+1$ points of color $i$, the result follows from Lemma 3 .

The total number of points in a $k$-colored set of points that satisfies the statement of Theorem [6 is $k^{2} n-k^{3}+k$. One can ask whether $n+o(n)$ points are sufficient to guarantee a curve complexity that does not depend on $n$. As the next theorem shows, this question has a negative answer for the case $k=2$.

Theorem 7. Let $c$ be a constant such that $c>1$. For every integer $n>2 \frac{c}{c-1}$ there exists a 2-colored planar graph $G=\left(V_{0} \cup V_{1}, E\right)$ and a 2-colored set of points $S=S_{0} \cup S_{1}$ consisting of $n+\frac{n}{c}$ points such that: (i) $\left|V_{0}\right|=\left|V_{1}\right|=\frac{n}{2}$; (ii) $\left|S_{0}\right|=\left|S_{1}\right|=\frac{n}{2}+\left\lceil\frac{n}{2 c}\right\rceil$; and (iii) any 2 -colored point-set embedding of $G$ on $S$ has one edge with at least $\frac{2}{3}\left\lfloor\frac{c-1}{2 c} n\right\rfloor-1$ bends. 


\section{$5.2 h$-Bend $k$-Colored Universal Grid}

Let $\mathcal{F}_{k}$ be the family of all $k$-colored planar graphs with $n$ vertices $(1 \leq k \leq n)$. An $h$-bend $k$-colored universal grid for $\mathcal{F}_{k}$ is a $k$-colored set of points $S$ such that: (i) $S$ is an integer grid; (ii) any element of $\mathcal{F}_{k}$ has a $k$-colored point-set embedding $\Gamma$ on $S$ with curve complexity at most $h$; and (iii) the bends of $\Gamma$ are at grid points. The drawing $\Gamma$ is called a $k$-colored point-set grid embedding. In this section we study the size of an $h$-bend $k$-colored universal grid that supports $k$-colored point-set grid embeddings whose curve complexity does not depend on the input size. Let $S$ be the $k$-colored set of points that contains points $p=(x, y)$ such that $x, y \in \mathbb{Z}$ and $0 \leq x, y<2 k N$ where $N=(n-k+1)(3 n-5)$. Let each point $p=(x, y)$ of $S$ have color $\operatorname{col}(p)=\left\lfloor\frac{x}{2 N}\right\rfloor$. We call $S$ the $(n, k)$-strip grid.

Theorem 8. Let $\mathcal{F}_{k}$ be the family of all $k$-colored planar graphs with $n$ vertices $(1 \leq k \leq n)$. The $(n, k)$-strip grid is a $(6 k+5)$-bend $k$-universal grid for $\mathcal{F}_{k}$. Furthermore, there exists an $\mathcal{O}(k n)$-time algorithm that computes a $k$-colored point-set grid embedding on $S$ of any $G \in \mathcal{F}_{k}$ with curve complexity at most $6 k+5$.

Sketch of Proof: Let $G$ be a $k$-colored planar graph with $n$ vertices and $m$ edges. Let $\sigma$ be an ordered $k$-colored sequence compatible with $G$ and such that elements colored $i$ appear before than elements colored $i+1(i=0, \ldots, k-2)$. By Theorem 36 admits an augmenting $k$-colored Hamiltonian path $\mathcal{H}$ consistent with $\sigma$. Since the $(n, k)$-strip grid $S$ contains more than $n$ points for each color we can arbitrarily choose a subset $S^{\prime}$ of $S$ such that $\operatorname{seq}(S)=\sigma$ and use Theorem 1 to compute a $k$-colored point-set embedding of $G$ on $S$. However, the technique behind Theorem 1 does not guarantee that the division vertices are at grid point even if the point in $S^{\prime}$ are grid points. We describe in the following a variant of this technique that places bends at grid points. Let $w_{0}, w_{1}, \ldots, w_{n^{\prime}}$ be the vertices (either division vertices or real vertices) of $\mathcal{H}$ in the order they appear in $\mathcal{H}$. Since $\sigma$ is ordered we have that the real vertices of $G$ in $\mathcal{H}$ are ordered along $\mathcal{H}$ except for the presence of the division vertices, i.e. if we ignore the division vertices then all vertices of the same color appear consecutively walking along $\mathcal{H}$. Define the following indices: $j_{i}=\max \left\{j \mid \operatorname{col}\left(w_{j}\right)=i\right\}$. All the division vertices $w_{j}$ such that $j_{i-1}<j<j_{i}$ are given color $i$, where we set $j_{-1}=-1$. With this coloring of the division vertices, we have that $\mathcal{H}$ is an ordered $k$-colored path, i.e. it consists of a set of vertices (either division vertices or real vertices) colored $c_{0}$, followed by a set of vertices colored $c_{1}$, etc. Since $\sigma$ has been chosen so that elements colored $i$ appear before than elements colored $i+1(i=0, \ldots, k-2)$, then $c_{i}=i(0 \leq i \leq k-1)$, i.e. $\mathcal{H}$ consists of a set of vertices colored 0 followed by a set of vertices colored 1 , etc. The number of real vertices of a given color $i$ is at most $n-k+1$ (because at least one vertex for any other color must exist), and the number of division vertices between a pair of consecutive real vertices is at most $m$ (because each edge of $\mathcal{H}$ is crossed at most once by an edge connecting two real vertices), which, in turn, is at most $3 n-6$ since the graph is planar. It follows that we have at most $(n-k+1)(m+1) \leq N$ vertices of each color. Let $w_{j}$ be a vertex (either a real or a division vertex) whose color is $i$, 
then $j_{i-1}<j \leq j_{i}$. Vertex $w_{j}$ is drawn at point having coordinates $(2 x, 2 x)$ where $x=i N+\left(j-j_{i-1}\right)-1$. Since $0 \leq 2\left(\left(j-j_{i-1}\right)-1\right)<2 N$, then $\left\lfloor\frac{2 x}{2 N}\right\rfloor=i$, i.e. point $(2 x, 2 x)$ is colored $i$. Let $e=\left(w_{j_{a}}, w_{j_{b}}\right)$ be an edge (either a real edge of $G$ or a portion of an edge of $G$ ). Since the endvertices of $\mathcal{H}$ are on the same face of $\operatorname{Ham}(G)$, there exists a planar embedding of $\operatorname{Ham}(G)$ such that $w_{0}$ and $w_{n^{\prime}-1}$ are on the external face. In such an embedding every edge not in $\mathcal{H}$ is either on the left-hand side of $\mathcal{H}$ or on the right-hand side of $\mathcal{H}$ when walking from $w_{0}$ to $w_{n^{\prime}-1}$. If $e$ belongs to $\mathcal{H}$, it is drawn as a straight-line segment. If $e$ is to the left of $\mathcal{H}$, then it is drawn with only one bend whose coordinates are $\left(2 x_{a}+1,2 x_{b}-1\right)$, where $\left(2 x_{a}, 2 x_{a}\right)$ is the point representing vertex $w_{j_{a}}$ and $\left(2 x_{b}, 2 x_{b}\right)$ is the point representing vertex $w_{j_{b}}$. If $e$ is to the right of $\mathcal{H}$, then it is drawn with only one bend whose coordinates are $\left(2 x_{b}-1,2 x_{a}+1\right)$.

Corollary 1. Every $n$-vertex planar graph admits a $k$-colored point-set grid embedding with curve complexity $6 k+5$ on a grid whose size is $\mathcal{O}\left(k n^{2}\right) \times \mathcal{O}\left(k n^{2}\right)$.

\section{References}

1. Badent, M., Di Giacomo, E., Liotta, G.: Drawing colored graphs on colored points. In: Dehne, F.K.H.A., Sack, J.-R., Zeh, N. (eds.) WADS 2007. LNCS, vol. 4619, pp. 102-113. Springer, Heidelberg (2007)

2. Di Battista, G., Eades, P., Tamassia, R., Tollis, I.G.: Graph Drawing. Prentice Hall, Upper Saddle River, NJ (1999)

3. Di Giacomo, E., Didimo, W., Liotta, G., Meijer, H., Trotta, F., Wismath, S.K.: kcolored point-set embeddability of outerplanar graphs. In: Kaufmann, M., Wagner, D. (eds.) GD 2006. LNCS, vol. 4372, pp. 318-329. Springer, Heidelberg (2007)

4. Di Giacomo, E., Didimo, W., Liotta, G., Wismath, S.K.: Curve-constrained drawings of planar graphs. Computational Geometry 30, 1-23 (2005)

5. Di Giacomo, E., Liotta, G., Trotta, F.: On embedding a graph on two sets of points. IJFCS, Special Issue on Graph Drawing 17(5), 1071-1094 (2006)

6. Kaneko, A., Kano, M.: Discrete geometry on red and blue points in the plane a survey. In: Discrete \& Computational Geometry, Algorithms and Combinatories, vol. 25, pp. 551-570. Springer, Heidelberg (2003)

7. Kaufmann, M., Wagner, D. (eds.): Drawing Graphs. LNCS, vol. 2025. Springer, Heidelberg (2001)

8. Kaufmann, M., Wiese, R.: Embedding vertices at points: Few bends suffice for planar graphs. Journal of Graph Algorithms and Applications 6(1), 115-129 (2002)

9. Pach, J., Wenger, R.: Embedding planar graphs at fixed vertex locations. Graph and Combinatorics 17, 717-728 (2001) 